\title{
Self-management programmes in temporomandibular disorders: results from an international Delphi process
}

J. DURHAM*, M. AL-BAGHDADI ${ }^{\dagger}$, L.BAAD-HANSEN ${ }^{*}$, M. BRECKONS ${ }^{\S}$,

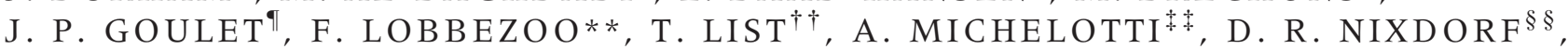

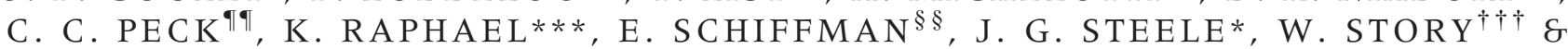
R. OHRBACH ${ }^{+1+}$ 'Centre for Oral Health Research $\theta$ Institute of Health and Society, Newcastle-upon-Tyne Hospitals' NHS Foundation Trust, Newcastle University, Newcastle-upon-Tyne, UK, ${ }^{\dagger}$ Oral Surgery Unit, Al-Noor Specialized Dental Care Centre, Iraqi Ministry of Health, Baghdad, Iraq, "Section of Orofacial Pain and Jaw Function, Institute of Odontology and Oral Health, Aarhus University, Aarhus, Denmark, ${ }^{\S}$ Institute of Health $\theta$ Society, Newcastle University, Newcastle-upon-Tyne, UK, " Faculty of Dental Medicine, Université Laval, Quebec, QC, Canada, **Department of Oral Health Sciences, Academic Centre for Dentistry Amsterdam (ACTA), MOVE Research Institute Amsterdam, University of Amsterdam and VU University Amsterdam, Amsterdam, The Netherlands, "†Department of Orofacial Pain and Jaw Function, Scandinavian Center for Orofacial Neurosciences (SCON), Faculty of Odontology, Malmö University, Malmö, Sweden, ${ }^{*}$ Section of Orthodontics, Department of Neuroscience, Reproductive and Oral Sciences, University of Naples Federico II, Naples, Italy, ${ }^{\S \S}$ Division of TMD and Orofacial Pain, School of Dentistry, University of Minnesota, Minneapolis, MN, USA, " The University of Sydney, Sydney, NSW, Australia, ${ }^{* * *}$ New York University College of Dentistry, New York, NY, USA, ${ }^{\dagger \dagger}$ Centre for Oral Health Research, Newcastle University, Newcastle-upon-Tyne, UK and "Wepartment of Oral Diagnostic Sciences, School of Dental Medicine, University at Buffalo, Buffalo, NY, USA

SUMMARY Self-management (SM) programmes are commonly used for initial treatment of patients with temporomandibular disorders (TMD). The programmes described in the literature, however, vary widely with no consistency in terminology used, components of care or their definitions. The aims of this study were therefore to construct an operationalised definition of self-management appropriate for the treatment of patients with TMD, identify the components of that self-management currently being used and create sufficiently clear and non-overlapping standardised definitions for each of those components. A four-round Delphi process with eleven international experts in the field of TMD was conducted to achieve these aims. In the first round, the participants agreed upon six principal concepts of self-management. In the remaining three rounds, consensus was achieved upon the definition and the six components of self-management. The main components identified and agreed upon by the participants to constitute the core of a SM programme for TMD were as follows: education; jaw exercises; massage; thermal therapy; dietary advice and nutrition; and parafunctional behaviour identification, monitoring and avoidance. This Delphi process has established the principal concepts of selfmanagement, and a standardised definition has been agreed with the following components for use in clinical practice: education; self-exercise; self-massage; thermal therapy; dietary advice and nutrition; and parafunctional behaviour identification, monitoring and avoidance. The consensus-derived concepts, definitions and components of SM offer a starting point for further research to advance the evidence base for, and clinical utility of, TMD SM.

KEYWORDS: temporomandibular disorders, temporomandibular joint disorders, self-care, selfmanagement, Delphi technique, Delphi process, conservative management

Accepted for publication 6 October 2016 


\section{Introduction}

The need and efficacy of initial, non-invasive, management for temporomandibular disorders (TMD) is long established $(1,2)$, and interest has recently increased in multimodal and 'tailored' approaches (35). A core part of non-invasive initial therapy is what is varyingly known as 'self-care' or 'self-management $(\mathrm{SM})^{\prime}$. Self-care or SM may be all that is required in those that are adaptive (copers) and self-motivated, or it may form part of a more complex multimodal treatment plan (4).

A recent systematic review (6) examined TMD selfcare and SM programmes and suggested grouping both under the term 'SM programmes', thereby ensuring consistency with the wider SM literature (7). Story et al. (6) highlighted the lack of an agreedupon definition of TMD SM and constructed a studyspecific definition for the review acknowledging its limitations.

Story et al. (6) identified 15 randomised controlled trials up to 15 April 2016 that fitted their inclusion criteria. In these 15 studies, there was heterogeneity in the components making up the SM programmes and also in the way the components were employed as behavioural change techniques. Although the SM components in the various SM programmes were not always employed in the same manner between studies, the most common groups of SM components were as follows: jaw (muscle) exercises, relaxation and posture training; relaxation strategies; education on TMD (and analgesia usage); self-monitoring advice for habits. The efficacy of SM as a stand-alone treatment was not established as it was usually the comparator treatment and was not compared to no treatment (or waiting list control), but the intra-group effect sizes for SM seemed promising and ranged from 0.3 to 3.1 (Cohen's d effect size). Perhaps unsurprisingly Story et al. called for a consensus-derived, operationalised definition of TMD SM in order to allow future research to clearly articulate the (behavioural) interventions employed under each of the components of SM, allow clinicians/researchers design their own SM programmes and examine their efficacy.

This study aims to (i) construct an operationalised definition of SM appropriate for the treatment of TMD, (ii) identify the components of SM, (iii) create sufficiently clear and non-overlapping definitions for each of those components through a Delphi process
(DP) with international experts in the field of TMD. It was not our intent to create operationalised definitions for each treatment component within SM given: the diversity of implementation of the components; the lack of understanding of the systems/processes underlying the therapeutic responses to each component; and data on their individual efficacy.

\section{Methods}

Estimation of the number of participants required for a DP varies dependent on purpose, and from the literature $(8,9)$, the minimum number of participants for this DP was set at seven; consequently, eleven international experts were initially invited by email to allow a one-third dropout rate.

The eleven TMD experts (LBH, JPG, TL, FL, AM, DN, RO, CP, KR, ES and JS) were identified from the literature and recent membership to international invitation-only TMD colloquia and workgroups. All had more than 10 years' experience in the field of TMD, with most having greater than thirty. All those approached agreed to participate in the study.

The first meeting (round 1) was held face-to-face at the International Association for Dental Research's annual meeting (Boston 2015). The International RDC/TMD (Research Diagnostic Criteria for Temporomandibular Disorders) Consortium Network hosted the meeting. Eleven of the study authors attended the first meeting (MAB, MB, JD, LBH, JPG, FL, AM, RO, $\mathrm{KR}, \mathrm{ES}$ and JS), and no expenses or incentives were offered. All participants received study-specific documentation ahead of the meeting: brief summary of the recently concluded systematic review on SM (6); outline of the areas for discussion during the DP. The areas for discussion initially included the following: (i) constructing an operationalised definition of SM, (ii) identifying the treatment components within SM and (iii) defining those components.

The first meeting was in the form of a focus group discussion, moderated by the first author (JD), and digitally recorded to allow cross-checking of the accuracy of the minutes on the definition of SM and its components (taken by $\mathrm{MB}$ ), which were then sent to all participants. All participants were asked to add further critique and revisions to the minutes document in an iterative process via email. The conclusion to each round was determined once all participants had added comments or indicated they had 
no further comments. Following each round, the first author collated all (responses to) comments and made revisions to the document. Comments and responses were displayed in the evolving document so that when it was re-circulated, individuals could track responses and revisions. When the revised document was ready, it was sent to all participants, drawing their attention to key areas requiring ongoing discussion, thereby signalling the start of the next round of discussions. This process continued until no new critique or comments were received (round four) on the revised document. All participants then helped construct and revise this manuscript as a final triangulation of the definition and components of SM emerging from the DP.

At the end of the process, SM programmes used by each participant were requested, translated to English where needed, and coded according to the SM components identified (MB coded, JD cross-checked validity) during the DP. The components of each participant's SM programme were summarised in a simple table to display the existing similarity in the written information on SM distributed to patients with TMD by the participants' institutions.

\section{Results}

As is to be expected in an active, iterative, DP discussions and critique varied by round of the Delphi process and for the sake of clarity, the presentation of the consensus reached will be summarised in two broad subheadings: principal concepts of SM; and definition of SM and the components comprising it. An overview of the content of each of the rounds of discussion of the Delphi process is shown in Fig. 1.

\section{Principal concepts of SM}

The first round of verbal discussions established and agreed the principal concepts of SM for the DP:

1 SM is a core part of TMD management.

$2 \mathrm{SM}$ is provided to the patient as a first essential step after diagnosis, but which can be built upon as necessary over time by different clinicians or specialities. SM is part of the continuing management of the patient with the intent that they will use it as needed throughout their life.

3 SM should be delivered verbally and supported with written information/instructions and should be appropriate for any clinical setting. Reinforcement might be possible using electronic media.

4 The decision over who delivers SM resides with the presiding clinician and the particular characteristics of the clinical facility where the patient is being managed. As there is a therapeutic nature to the doctor/patient relationship ('therapeutic alliance') (10), there are different models of provision. SM does not necessarily need to be delivered by a specialist or a clinician (5).

5 SM contains context-dependent education, including explanation, advice and reassurance (e.g. optimistic counselling). Such education can be provided, at least in part, even if a definitive diagnosis is not reached, and can include generic information on the nature of persistent pain and painrelated TMD subject to no 'red flags' being present, such as sensory or motor function changes.

$6 \mathrm{SM}$ can be reviewed to ensure comprehension and adherence. Follow-up could be face-to-face or by telephone contact. Further research is required to determine the optimum mode and time for review.

The definition of SM and the components comprising it took three further rounds to clarify and establish consensus with the group recognising the difficulty in prescribing a gold-standard programme, or contents list for specific interventions under each treatment component of SM due to the paucity of evidence on the efficacy of one particular intervention over another. Instead, the group elected to define the core components of SM, which then would guide individuals as to the construction of future SM programmes and trials of particular interventions under each component.

\section{Definition of SM and the components comprising it}

The definition of SM proved difficult to construct due to the absence of data or definitions in the literature regarding SM (6). In contrast, opinions on the components comprising SM were largely similar. The areas requiring most discussion to establish a consensus on a definition of SM were as follows: the specificity of the aims of SM; whether intra-oral appliances could be considered a component of SM; and whether analgesia (of any form) could be considered a component of SM.

The specificity of the aims of SM was a matter for extensive discussion because the need for SM to be applicable to any subtype of TMD (11). For example, the aims of a potentially goal-oriented SM programme 


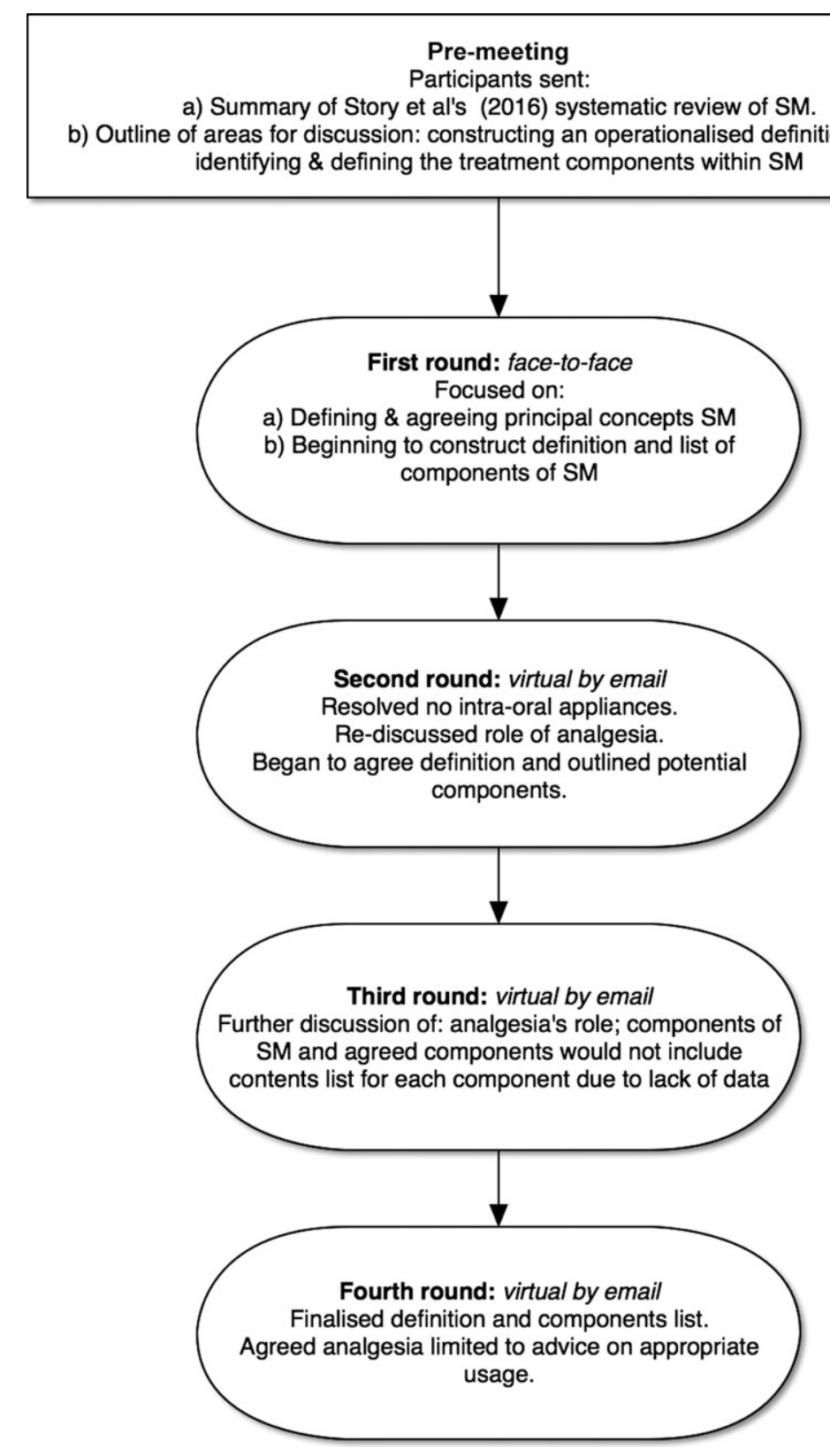

Fig. 1. Flow chart of rounds of the Delphi process summarising key actions at each round. SM, selfmanagement.

for a patient with limited mouth opening due to a disc displacement without reduction with limited opening were contrasted in our discussion to the aims of a programme for myalgia.

Over the period of discussion, it was agreed, however, that there were common, but generic, aims for all SM programmes. It was also agreed that these aims could be supplemented by further objectives and adjunctive modules (treatments) specific to the individual's presenting complaint, for example a more goal-oriented programme to address limited mouth opening. Similarly, the order of components in the
SM programme should be tailored to the individual's complaint. The components of a standard core SM programme were therefore agreed through consensus as those shown in Table 1, and the final definition of SM was as follows:

SM programmes in TMD are a core component of management of TMD throughout its course. SM programmes are defined as a group of procedures that have a logical basis for therapeutic action in relation to the respective diagnosis for 
Table 1. Components of standard core SM programme

\begin{tabular}{|c|c|}
\hline Component & Agreed definition of component \\
\hline Education & $\begin{array}{l}\text { Education pertains to diagnosis and generally favourable prognosis ('optimistic counselling'), } \\
\text { when appropriate, and it includes reassurance that TMD is typically a benign group of conditions } \\
\text { and self-limiting in the vast majority of cases, but can fluctuate in symptomatology. This education } \\
\text { should also include TMD's biopsychosocial aetiology and its limited sequelae in the twelve most } \\
\text { common subtypes* and cautions against invasive and irreversible treatments. } \\
\text { Other areas of education where basic information should be included are as follows: sleep practices; } \\
\text { sensible and time-limited use of analgesia; avoidance of OTC splints bought without consultation } \\
\text { with dentist; caffeine usage; 'doctor shopping'; anatomy and usual function of TMJ complex and } \\
\text { associated musculature. For example, making the patient aware of the masticatory anatomy and why it } \\
\text { is painful; and also clicking sounds and why it is a painful click ensuring patients do not become } \\
\text { hypervigilant or 'play' with their lower jaw to constantly check for TMJ noise; warning against extensive } \\
\text { occlusal adjustments and invasive interventions without clear-cut, objective signs of TMJ pathology. } \\
\text { In definite, predominant, arthrogenous complaints, surgical interventions should be explained in a } \\
\text { neutral manner explaining their risks, the poor evidence base, and the fact that they are not proven to } \\
\text { be superior to simple, non-invasive interventions, which carry fewer risks }\end{array}$ \\
\hline Self-exercise therapy & $\begin{array}{l}\text { This was not labelled as physical therapy as the participants felt that 'exercise therapy' or 'physical } \\
\text { therapy' would be misleading. The focus in SM is on self-applied exercises by the patient vs. manual } \\
\text { therapies performed by healthcare professionals. Exercises can be implemented by the clinician or by } \\
\text { referral to other healthcare professions including physical or occupational therapists }\end{array}$ \\
\hline Thermal modalities & Use of heat and/or ice to areas of pain \\
\hline Self-massage therapy & $\begin{array}{l}\text { Massage is limited to the anatomic location of the painful or tense affected masticatory muscles } \\
\text { (most easily accessible to palpation are masseter muscle and temporalis muscle) }\end{array}$ \\
\hline Diet and Nutrition & $\begin{array}{l}\text { Specific nutritional advice is available in the Temporomandibular Joint Association's (TMJA) guide to } \\
\text { nutrition freely available online. Another aspect of this pertains to chewing restriction, which is to be } \\
\text { phrased as a 'pain-free diet', as opposed to a 'soft diet', with the recommendation that it be implemented } \\
\text { for a 2-week period, following which a review determines whether the individual advances as tolerated } \\
\text { to firmer and chewier consistency foods. This was phrased in this way because of concerns of } \\
\text { misinterpretation and therefore prolonged use of a soft diet }\end{array}$ \\
\hline Parafunctional behaviour & $\begin{array}{l}\text { This component should orient the patient towards identification, monitoring, and avoidance of any } \\
\text { parafunctional behaviour that exacerbate their pain. It was agreed that all three elements are important } \\
\text { as far as definition for an SM programme is concerned. The therapeutic boundaries of this component in } \\
\text { terms of self-identification of behaviours and control via avoidance, vs. formal cognitive-behavioural } \\
\text { therapy (CBT) (for example), are yet to be determined }\end{array}$ \\
\hline
\end{tabular}

*Twelve most common types of TMD (11): Myalgia; Local myalgia; Myofascial pain; Myofascial pain with referral; Arthralgia; Disc displacement with reduction; Disc displacement with reduction with intermittent locking; Disc displacement without reduction with limited opening; Disc displacement without reduction without limited opening; Degenerative joint disease; Subluxation; Headache attributable to TMD.

which they are recommended. The procedures comprising SM should be simple enough to allow patients to be readily instructed in their execution and to retain control of their execution. At the initial point of diagnosis, the patient should be given a core SM programme that can be built upon over time, as necessary, dependent on the clinical findings and course. The core SM programme for TMD consists of the following components: education; exercise; selfmassage; thermal therapy; dietary advice and nutrition; and parafunctional behaviour identification, monitoring and avoidance. Each component of SM presumably has a different mechanism of action, but in general, the main aim of SM is to allow healing and prevent further injury to the musculoskeletal system. SM programmes should not focus solely on physical symptoms but should also adopt a biopsychosocial approach and facilitate a return to normal function. SM programmes rely on a therapeutic alliance between clinician and patient and therefore require patient comprehension, motivation, cooperation, active participation, and adherence as well as clinician commitment, monitoring and titration. It empowers the 
patient to know that they have the innate ability to heal and the clinician engaging them and training them to do SM facilitates this.

Intra-oral appliances were discussed in the first two rounds resulting in a consensus that these were a separate management technique to SM because SM without intra-oral appliances was efficacious and therefore did not mandate the use of an intra-oral appliance. It was noted during discussions that as part of the education in SM, patients should be fully informed about advantages/disadvantages (complications) of intra-oral appliances; for example, it is appropriate to advise caution regarding the use of over-the-counter intra-oral appliances if not prescribed by a dental professional (12).

Analgesia was discussed throughout all rounds, specifically in relation to prescribing simple analgesia such as non-steroidal anti-inflammatory drugs and over-the-counter (OTC) analgesia usage and their potential adverse effects if used long term. The consensus reached was that in SM, individuals should receive specific education on time-limited use of appropriate OTC analgesia as well as its potential to cause complications, for example medication overuse headache, and that the prescribing of analgesia is a different form of management to SM. The group acknowledged that in one study SM with medication had a better short-term outcome than SM alone for jaw muscle pain (13). This effect, may, however depend on the type of muscle pain (14), and medication's usage has the undesired potential for patients to believe that the medication, and not the SM, has reduced their pain.

Table SI displays the components used by each participant's institution in their written SM documentation for patients.

\section{Discussion}

This DP has produced an expert-based standardised definition of SM and its components thereby providing a foundation for future research into the effectiveness of these recommendations. Each component of the SM programme was defined, but not operationalised (Table 1) in recognition that, if we consider various manners in which the components are implemented by just the members of this study (Table S1), we are currently uncertain whether there may be core elements of a given SM component that demand operationalisation in particular ways. We also do not fully understand whether there is, for example, a systems process involved, such that any form of SM carries with it substantial psychological benefits that are perhaps more responsible for any therapeutic response. Consequently, we have intentionally left the particular operationalisation of the specific SM components open for discovery. At the same time, the present paper provides a framework by which the reporting and interpretation of trials utilising SM programmes can be more carefully aligned.

The DP also highlighted significant gaps in the knowledge base on SM for TMD. Although it is widely accepted as a first-line efficacious management technique, there are sparse data on what interventions within each component of SM are most efficacious (6). Further to this, it is difficult to determine the modifications to SM required for particular TMD subtypes.

It is unlikely SM components exist as isolated forms of therapy; rather, self-regulatory behaviours, such as those underlying successful SM, exert effects at varying levels within the individual (15) and exert effects on overall pain processing. Dietary and nutritional guidance in TMD has been highlighted as an area of difficulty in TMD management (16) with recent improvements (http://tmj.org/common/file?id=179, last accessed 18 July 2016). There are, however, critical questions regarding dietary functioning left unanswered and may link to other pain disorder constructs for example should the patient adjust the texture of their diet for a period of time (and for how long?) in order to reduce their pain, or does chewing restriction encourage self-perpetuating fear-avoidance behaviour and therefore should we encourage graded masticatory exercise (17)? The anecdotal suggestion from this group was for recommending a review after 2 weeks to assess function and consider whether or not a graded return to normal texture foods as tolerated could be implemented, assuming that other SM components are concurrently being successfully implemented. Despite the absence of evidence, it is likely that any period of chewing restriction impacts beyond the observed respondent pain associated with mastication, highlighting the importance of better understanding each SM component's mechanisms and implementation.

Parafunctional activity raises different questions relating to SM and the implementation of what might be complex behaviour treatments. Reliable assessment of parafunctional activity and its role in TMD is a source of speculation and discussion (18-20). Recent 
advances have been made in to establish standards for parafunctional activity assessment and its potential role in $\operatorname{TMD}(21,22)$. The role of the parafunctional behaviour identification, monitoring and avoidance component of SM was discussed at length. Current evidence suggests that parafunctional activities must at least pervade through everyday life to play a role in TMD $(21,23)$. Simultaneously, other available evidence indicates a complex relationship between parafunction and pain (24-26). Consequently, one aspect of SM should be therefore to carefully evaluate the potential causal relationships between presumed parafunction and the patient's pain, and to proceed with the treatment aspect of this component of SM only with carefully gathered behaviour data and symptom reports. We suspect that other areas of SM, for example jaw exercises, will exhibit similar research questions and challenges. Prospective research would benefit by attending to these complex matters.

The preceding descriptions of different aspects of an SM programme illustrate that SM may not be as simple as it appears. In addition, many, if not all, of the components proposed here in the SM programme for TMD have little to no supporting evidence for their particular efficacy in TMD, and consequently, we have little to no empirical guidance regarding how to best employ SM and for which patients. For example, when should we use hot versus cold (and for what duration, and how many times a day); try to control parafunctional behaviours (and how do we do it reliably). Yet, this is precisely why this study was initiated: to standardise the concept of SM, identify all components of SM and provide initial definitions. From this beginning, it becomes possible to conduct research that can systematically examine different ways of operationalising SM components. For example, the SM component of parafunctional behaviour identification, monitoring and avoidance assumes that it is possible for patients to identify and modify such behaviours within the context of the SM programme containing other components. However, because these waking behaviours are often difficult to detect (27), it may be premature to include the parafunction component into an SM programme in contrast to addressing these behaviours via formal therapy, by a mental health professional, including cognitive-behavioural therapy. If identification, monitoring and avoidance can be performed, does their modification exert a therapeutic effect proportional to the effort sometimes required of the patient in adhering to this part of an SM programme? From a clinical perspective, this singular example points to the challenge for SM: initial diagnosis is only the starting point, and ongoing evaluation and critical testing of clinical hypotheses must accompany SM if it is to be as successful.

In summary, this DP has established an international consensus regarding the principal concepts of SM and a standardised definition of SM and its components. Future research is required to examine the validity and applicability of these consensus-derived concepts, definitions and components. Prospective research is required to evaluate cause-effect relationships between presumed causative or contributing factors, patient's complaints, and their response to each individual component of SM and the whole SM programme. Future trials also need to investigate the efficacy of different types and/or techniques of particular interventions under each individual component of SM against each other for management of particular subtypes of TMD. This will help in developing evidence-based SM programmes and assist in establishing operationalised definitions of SM components. Furthermore, research needs to explore the best modes of SM delivery and SM review including the appropriate time of SM delivery and proper period to review SM therapeutic outcomes as well as the use of electronic reinforcement of SM.

\section{Conclusion}

A standardised definition of SM and its components is now available for use in clinical practice. This definition and the principal concepts of SM agreed during the DP should allow the evidence base to be expanded in a more homogenous, comparable, manner in order to advance the science behind SM of TMD.

\section{Disclosure}

The authors have no conflict of interest to declare.

\section{Acknowledgments}

This study was unfunded, but the authors would like to acknowledge and thank the International Association for Dental Research and the International RDC/TMD (Research Diagnostic Criteria for Temporomandibular Disorders) Consortium Network for helping host the meeting. This study did not require ethical approval. 


\section{References}

1. Greene CS. Managing the care of patients with temporomandibular disorders: a new guideline for care. J Am Dent Assoc. 2010;141:1086-1088.

2. Greene CS. The etiology of temporomandibular disorders: implications for treatment. J Orofac Pain. 2001;15:93-105; discussion 106.

3. Kotiranta U, Suvinen T, Forssell H. Tailored treatments in temporomandibular disorders: where are we now? A systematic qualitative literature review. J Oral Facial Pain Headache. 2014;28:28-37.

4. Türp JC, Jokstad A, Motschall E, Schindler HJ, WindeckerGétaz I, Ettlin DA. Is there a superiority of multimodal as opposed to simple therapy in patients with temporomandibular disorders? A qualitative systematic review of the literature. Clin Oral Implant Res. 2007;18:138-150.

5. Dworkin SF, Huggins KH, Wilson L, Mancl L, Turner J, Massoth D et al. A randomized clinical trial using research diagnostic criteria for temporomandibular disorders-axis II to target clinic cases for a tailored self-care TMD treatment program. J Orofac Pain. 2002;16:48-63.

6. Story WP, Durham J, Al-Baghdadi M, Steele J, AraujoSoares V. Self-management in temporomandibular disorders: a systematic review of behavioural components. J Oral Rehabil. 2016;43:759-770.

7. Lorig KR, Holman H. Self-management education: history, definition, outcomes, and mechanisms. Ann Behav Med. 2003;26:1-7.

8. Cramer CK, Klasser GD, Epstein JB, Sheps SB. The Delphi process in dental research. J Evid Based Dent Pract. 2008;8:211-220.

9. Hsu CC, Sandford BA. The Delphi technique: making sense of consensus. Pract Assess. 2007;12:1-8.

10. Laskin DM, Greene CS. Influence of the doctor-patient relationship on placebo therapy for patients with myofascial pain-dysfunction (MPD) syndrome. J Am Dent Assoc. 1972;85:892-894.

11. Schiffman E, Ohrbach R, Truelove E, Look J, Anderson G, Goulet J-P et al. Diagnostic criteria for temporomandibular disorders (DC/TMD) for clinical and research applications: recommendations of the International RDC/TMD Consortium Network* and Orofacial Pain Special Interest Group. J Oral Facial Pain Headache. 2014;28:6-27.

12. Wassell RW, Verhees L, Lawrence K, Davies S, Lobbezoo F. Over-the-counter (OTC) bruxism splints available on the Internet. Br Dent J. 2014;216:E24.

13. Herman CR, Schiffman EL, Look JO, Rindal DB. The effectiveness of adding pharmacologic treatment with clonazepam or cyclobenzaprine to patient education and selfcare for the treatment of jaw pain upon awakening: a randomized clinical trial. J Orofac Pain. 2002;16:64-70.

14. Alencar FGJ, Viana PG, Zamperini C, Becker A. Patient education and self-care for the management of jaw pain upon awakening: a randomized controlled clinical trial comparing the effectiveness of adding pharmacologic treatment with cyclobenzaprine or tizanidine. J Oral Facial Pain Headache. 2014;28:119-127.
15. Bandura A. The primacy of self-regulation in health promotion. Appl Psychol. 2005;54:245-254.

16. Durham J, Touger-Decker R, Nixdorf DR, Rigassio-Radler D, Moynihan P. Oro-facial pain and nutrition: a forgotten relationship? J Oral Rehabil. 2015;42:75-80.

17. Vlaeyen JWS, Linton SJ. Fear-avoidance and its consequences in chronic musculoskeletal pain: a state of the art. Pain. 2000;85:317-332.

18. Raphael KG, Sirois DA, Janal MN, Wigren PE, Dubrovsky B, Nemelivsky LV et al. Sleep bruxism and myofascial temporomandibular disorders. J Am Dent Assoc. 2012;143:12231231.

19. Raphael KG, Janal MN, Sirois DA, Dubrovsky B, Klausner JJ, Krieger AC et al. Validity of self-reported sleep bruxism among myofascial temporomandibular disorder patients and controls. J Oral Rehabil. 2015;42:751-758.

20. Marbach JJ, Raphael KG, Janal MN, Hirschkorn-Roth R. Reliability of clinician judgements of bruxism. J Oral Rehabil. 2003;30:113-118.

21. Ohrbach R, Fillingim RB, Mulkey F, Gonzalez Y, Gordon S, Gremillion $\mathrm{H}$ et al. Clinical findings and pain symptoms as potential risk factors for chronic TMD: descriptive data and empirically identified domains from the OPPERA case-control study. J Pain. 2011;12:T27-T45.

22. Lobbezoo F, Ahlberg J, Glaros AG, Kato T, Koyano K, Lavigne GJ et al. Bruxism defined and graded: an international consensus. J Oral Rehabil. 2013;40:2-4.

23. Ohrbach R, Bair E, Fillingim RB, Gonzalez Y, Gordon SM, Lim PF et al. Clinical orofacial characteristics associated with risk of first-onset TMD: the OPPERA prospective cohort study. J Pain. 2013;14:T33-T50.

24. van der Meulen MJ, Lobbezoo F, Aartman IHA, Naeije M. Validity of the oral behaviours checklist: correlations between OBC scores and intensity of facial pain. J Oral Rehabil. 2013;41:115-121.

25. Glaros AG, Tabacchi KN, Glass EG. Effect of parafunctional clenching on TMD pain. J Orofac Pain. 1998;12:145-152.

26. Glaros AG, Williams K, Lausten L. Diurnal variation in pain reports in temporomandibular disorder patients and control subjects. J Orofac Pain. 2008;22:115-121.

27. Kaplan S, Ohrbach R. Self-report of waking-state oral parafunctional behaviors in the natural environment. J Oral Facial Pain Headache. 2016;30:107-119.

Correspondence: Justin Durham, Centre for Oral Health Research \& Institute of Health $\&$ Society, School of Dental Sciences, Framlington Place, Newcastle University, Newcastle-upon-Tyne NE2 4BW, UK. E-mail: justin.durham@ncl.ac.uk

\section{Supporting Information}

Additional Supporting Information may be found in the online version of this article:

Table S1 Self-management programmes by clinical setting. 